\title{
Effects of Neuregulin 3 Genotype on Human Prefrontal Cortex Physiology
}

\author{
Heike Tost, ${ }^{1,2}$ Joseph H. Callicott, ${ }^{1}$ Roberta Rasetti, ${ }^{1}$ Radhakrishna Vakkalanka, ${ }^{1,3}$ Venkata S. Mattay, ${ }^{1,3}$ \\ Daniel R. Weinberger, ${ }^{1,3,4 *}$ and Amanda J. Law ${ }^{1,5 *}$ \\ ${ }^{1}$ Clinical Brain Disorders Branch, Genes, Cognition, and Psychosis Program, National Institute of Mental Health, National Institutes of Health, Department \\ of Health and Human Services, Bethesda, Maryland 20892, ${ }^{2}$ Department of Psychiatry and Psychotherapy, Central Institute of Mental Health, Medical \\ Faculty Mannheim, University of Heidelberg, 61859 Mannheim, Germany, ${ }^{3}$ Lieber Institute for Brain Development, Johns Hopkins University Medical \\ Campus, Baltimore, Maryland 21205, ${ }^{4}$ Departments of Psychiatry, Neurology, and Neuroscience and McKusick-Nathans Institute of Genetic Medicine, \\ Johns Hopkins School of Medicine, Baltimore, Maryland 21205, and 5 Departments of Psychiatry and Cell and Developmental Biology, University of \\ Colorado, School of Medicine, Aurora, Colorado 80045
}

The neuregulin 3 gene (NRG3) plays pleiotropic roles in neurodevelopment and is a putative susceptibility locus for schizophrenia. Specifically, the T allele of NRG3 rs 10748842 has been associated with illness risk, altered cognitive function, and the expression of a novel splice isoform in prefrontal cortex (PFC), but the neural system effects are unexplored. Here, we report an association between rs 10748842 and PFC physiology as measured by functional magnetic resonance imaging of human working memory performance, where a convincing link between increased genetic risk for schizophrenia and increased activation in some PFC areas has been established. In 410 control individuals (195 males, 215 females), we detected a highly significant effect of NRG3 genotype manifesting as an unanticipated increase in ventrolateral PFC activation in nonrisk-associated $\mathrm{C}$ allele carriers. An additional analysis including 78 patients with schizophrenia spectrum disorders (64 males, 14 females) and 123 unaffected siblings ( 53 males, 70 females) revealed a whole-brain significant genotype by group interaction in right dorsolateral PFC (DLPFC), manifesting as a relative activation increase in healthy controls and siblings $(\mathrm{C}>\mathrm{T} / \mathrm{T})$ and as a hypoactivation in patients $(\mathrm{T} / \mathrm{T}>\mathrm{C})$. These observed genotype-dependent effects in PFC were not explained by task performance and did not conform to established locales of prefrontal inefficiency linked to genetic risk for schizophrenia. Our data indicate a complex modulation of brain physiology by rs10748842, which does not fit the simple inefficiency model of risk association in DLPFC and suggests that other neurobiological mechanisms are involved.

Key words: ErbB4; genetics; Neuregulin; neuroimaging; NRG3; schizophrenia

\section{Introduction}

Neuregulin 3 (NRG3) is a neuronal-enriched growth factor and a specific ligand for the ErbB4 receptor kinase that plays pleiotropic roles in brain development and plasticity. Similar to its paralogue NRG1, it is central for the development of the embryonic cerebral cortex by regulating the proliferation, differentiation, and migration of neural progenitor cells (Carteron et al., 2006). Several studies have linked genetic variation in the NRG3 gene (NRG3) to a heterogeneous group of neurodevelopmental disorders, including developmental delay, cognitive impairment, and

Received Aug. 13, 2013; revised Nov. 22, 2013; accepted Nov. 27, 2013.

Author contributions: V.S.M., D.R.W., and A.J.L. designed research; H.T., J.H.C., R.V., V.S.M., D.R.W., and A.J.L. performed research; H.T., J.H.C., R.R., V.S.M., D.R.W., and A.J.L. analyzed data; H.T., D.R.W., and A.J.L. wrote the paper.

This work was supported by funds from the Intramural Research Program of the National Institute of Mental Health, National Institutes of Health.

This manuscript is in memory of Dr. Radhakrishna Vakkalanka.

*D.R.W. and A.J.L. contributed equally to this work.

The authors declare no competing financial interests.

Correspondence should be addressed to Dr. Amanda J. Law, University of Colorado, School of Medicine, Mailstop 8344, RC1 North, RM. 8101, Aurora, C0 80045. E-mail: amanda.law@ucdenver.edu.

DOI:10.1523/JNEUROSCI.3496-13.2014

Copyright $\odot 2014$ the authors $\quad 0270-6474 / 14 / 341051-06 \$ 15.00 / 0$ psychiatric illness (Balciuniene et al., 2007). In particular, evidence has accumulated for an association of NRG3 with schizophrenia. To date, several linkage studies have implicated the genomic region of NRG3 (10q22-q23) as a schizophrenia susceptibility locus (Fallin et al., 2003; Faraone et al., 2006; Xu et al., 2009). Fine mapping of NRG3 has identified genome-wide significant association between delusion severity and polymorphisms in an ultraconserved region of linkage disequilibrium in intron 1, proxied by rs10748842 (Chen et al., 2009). In our prior work (Kao et al., 2010), we confirmed the association of rs10748842 with schizophrenia and demonstrated that a potential molecular mechanism of risk involves elevated expression of a novel NRG3 splice isoform in the prefrontal cortex (PFC). Emerging support also comes from behavioral studies demonstrating that variants in allelic identity with rs 10748842 impact cognition in schizophrenia patients and healthy controls (Morar et al., 2011; Meier et al., 2013).

In recent years, the investigation of genetic associations with neuroimaging phenotypes has emerged as a popular research strategy for elucidating neural system mechanisms of established clinical genetic associations, partly because of the prediction that genes are more penetrant at the level of brain physiology than at 
clinical diagnosis (Hariri and Weinberger, 2003). This implicates that risk-associated alterations in brain function can be identified in healthy risk allele carriers and that the phenotype can be compared with that of a clinical sample even if the examined variant is not statistically enriched in patients. The approach aids in the search for disease mechanisms provided that a given phenotype is heritable and its link to increased genetic risk for the illness has been convincingly established, e.g., by evidence of similar abnormalities in healthy relatives of affected patients (Rasetti and Weinberger, 2011). A well characterized example is alterations in dorsolateral PFC (DLPFC) activation during working memory, which has been reliably observed in schizophrenia patients, their unaffected first-degree relatives, and healthy carriers of genetic risk variants for the disorder (Rasetti and Weinberger, 2011), whereby greater activation of the DLPFC (for a fixed level of performance) has been identified as an indicator of prefrontal physiological inefficiency related to genetic risk (Callicott et al., 2003a). DLPFC [Brodmann area (BA) 46 and 9] and ventrolateral PFC (VLPFC; BA 44 and 45) play different roles in spatial working memory, with DLPFC controlling the maintenance and manipulation of working memory contents and VLPFC assisting in content selection and the inhibition of irrelevant information (Aron et al., 2003; Koechlin et al., 2003).

Here, we report an association between rs10748842 and brain physiology as measured by functional magnetic resonance imaging ( $\mathrm{fMRI}$ ) examination of working memory performance, an established intermediate phenotype for schizophrenia that maps to the DLPFC (Callicott et al., 2003a). We hypothesized that the $N R G 3$ risk-associated genotype would map to DLPFC regions of inefficiency associated with increased risk for schizophrenia, as observed previously for other genes [e.g., GRM3 (Egan et al., 2004), KCNH2 (Huffaker et al., 2009), miR-137 (van Erp et al., 2013)]. We observed strong effects of rs 10748842 on VLPFC and DLPFC function; however, they did not map to regions showing an association with increased genetic risk. Our results suggest a complex relationship between NRG3, schizophrenia, and prefrontal cortical function.

\section{Materials and Methods}

Subjects. Participating subjects were recruited as part of the NIMH Clinical Brain Disorders Branch "Sibling Study," an ongoing investigation of neurobiological abnormalities related to the genetic risk for schizophrenia (protocol 95-M-0150; principal investigator, Daniel R. Weinberger). A total of 611 subjects were studied: 410 healthy volunteers (195 males, 215 females), 78 individuals with Diagnostic and Statistical Manual of Mental Disorders, fourth revision (DSM-IV) schizophrenia spectrum disorder (64 males, 14 females), and 123 healthy siblings of schizophrenia patients (53 males, 70 females). Only Caucasians of self-identified European descent were studied to minimize population stratification artifacts. All participating subjects were $18-55$ years of age, above 75 in IQ, and able to give informed consent. Other specific exclusion criteria included significant medical or neurological problems, history of head trauma, and prior alcohol or drug abuse. Structured clinical interviews for DSM-IV were acquired by qualified raters, including research psychiatrists, to verify the presence or absence of a lifetime history of psychiatric illness. Diagnostic discrepancies were resolved in conference. None of the healthy volunteers had a first-degree relative with a schizophrenia spectrum disorder or received psychotropic pharmacological treatment. Patients with schizophrenia were on a stable regimen of antipsychotic medication (classical neuroleptics or atypical antipsychotics). None of the siblings had a current or past psychiatric history of schizophrenia spectrum disorders or received psychotropic pharmacological treatment. All participants provided written informed consent for a protocol approved by the NIMH Institutional Review Board.
Genotyping procedures. We used standard methods to extract DNA from white blood cells with the Puregene DNA purification kit (Gentra Systems). Genotypes for single-nucleotide polymorphisms in the first intron of NRG3 (rs10748842 and rs6584400) were determined with the Taqman allelic discrimination assay (details available on request). The observed genotype distributions did not deviate from Hardy-Weinberg equilibrium $\left(\chi^{2}=4.31, p>0.1\right)$. As expected (Kao et al., 2010; Morar et al., 2011), rs10748842 and rs6584400 genotypes proved to be in near allelic identity $\left(\mathrm{D}^{\prime}=0.97, r^{2}=0.73 p<0.001\right)$. Thus, rs10748842 genotypes were used as a proxy for both markers in subsequent imaging genetics analyses. Based on our previous expression work on this variant showing differences in expression between C carriers and TT homozygote individuals (Kao et al., 2010), and on the low frequency of the rs $10748842 \mathrm{C}$ allele in Caucasians (0.09), C/C and C/T individuals were merged for all analyses. Genotype groups did not significantly differ with respect to age, sex, education, handedness, and task performance. Moreover, in patients, no genotype-dependent differences were observed in measures of chlorpromazine equivalents (CPZEs), symptom severity, and global functioning. Patient genotype groups did differ in measures of IQ, a variable controlled for in subsequent fMRI analyses. Details on the subjects' demographics and task performance stratified by NRG3 genotype and diagnosis are provided in Table 1.

$\mathrm{N}$-back working memory task. Brain function was studied with fMRI and a well established $n$-back working memory paradigm that robustly engages the prefrontal cortex (Callicott et al., 2003a; Tan et al., 2006; Nixon et al., 2011). In this block-designed task, a series of visual stimuli (numbers one to four) are displayed on a screen in a random order at set locations in a diamond-shaped box (fixed stimulus presentation time, $500 \mathrm{~ms}$; interstimulus interval, $1500 \mathrm{~ms}$ ). Participants responded to each stimulus via a MRI-compatible button box with four buttons arranged in the same configuration as the stimuli presented on the screen. In the 2-back condition, subjects were asked to encode the currently seen number, simultaneously recall the number seen two presentations previously, and press the button corresponding to the position of the number two presentations previously. During the control condition ( 0 -back) subjects were asked to press the button corresponding to the position of the current number presentation. The task was presented in eight blocks of $30 \mathrm{~s}$ each, with alternating epochs of 0 -back and 2-back conditions.

fMRI data acquisition and processing. fMRI data were acquired on a 3 tesla whole-body scanner (GE Signa) using a gradient-echo echoplanar imaging sequence with the following specifications: 24 axial slices; $6 \mathrm{~mm}$ thickness; voxel dimension, $3.75 \times 3.75 \mathrm{~mm}$; TR, $2000 \mathrm{~ms}$; TE, $30 \mathrm{~ms} ; 90^{\circ}$ flip angle; $24 \mathrm{~cm}$ field of view; $64 \times 64$ matrix. Images were processed as detailed previously (Rasetti et al., 2011) using standard procedures in SPM5 (http://www.fil.ion.ucl.ac.uk/spm/). Briefly, all images were realigned to the first image of the scan run, spatially normalized into standard Montreal Neurological Institute (MNI) stereotactic space, and smoothed with an $8 \mathrm{~mm}$ full-width half-maximum Gaussian filter. Quality control included visual inspection of the data and the analysis of parameter estimates for ghosting artifacts, signal-to-noise ratios, signal variance, and head motion.

Data analysis. The processed images were analyzed in a two-level procedure. At the first level, separate general linear models were specified for each subject by modeling the alternating task conditions as a box-car reference vector that was convolved with the SPM5 standard hemodynamic response function. The six head-motion parameters from the realignment step were included as nuisance covariates into the model. At the model estimation stage, the data were high-pass filtered with a cutoff of $128 \mathrm{~s}$, and an autoregressive model was applied. Individual linear contrast images of the 2-back $>0$-back conditions were subsequently computed and entered in second-level group analyses. Specifically, the effects of NRG3 genotype were examined in a summary statistic procedure using general linear models in SPM5 with random-effects group statistics as detailed previously (Rasetti et al., 2011). We first tested for a simple main effect of NRG3 rs10748842 genotype on prefrontal physiology in healthy volunteers using a multiple regression model with NRG3 genotype (T/T homozygotes, $\mathrm{C}$ allele carriers) as a covariate of interest and age, sex, and $n$-back task performance (percentage correct responses) as nuisance covariates. Then, to explore possible genotype by 
Table 1. Subjects' demographics and task performance by rs10748842 genotype

\begin{tabular}{|c|c|c|c|}
\hline & $T / T$ & C carriers & $p$ value \\
\hline \multicolumn{4}{|l|}{ Normal controls $(n=410)$} \\
\hline Number & 326 & 84 & \\
\hline Sex: males/females & $156 / 170$ & $39 / 45$ & 0.46 \\
\hline Age: mean (SD), years & $31.2 \mid(8.9)$ & $31.9 \mid(9.7)$ & 0.49 \\
\hline Education: mean (SD), years & $17.0 \mid(2.5)$ & $16.9 \mid(2.8)$ & 0.95 \\
\hline Handedness: mean (SD) & $78.1(46.8)$ & $78.4 \mid(46.9)$ & 0.96 \\
\hline IQ: mean (SD) & $109.1 \mid(9.4)$ & $109.6 \mid(8.2)$ & 0.63 \\
\hline 2-back correct: mean (SD), \% & $84 \mid(15)$ & $80 \mid(19)$ & 0.11 \\
\hline 2-back RT: mean (SD), seconds & $0.49 \mid(0.23)$ & $0.50 \mid(0.24)$ & 0.54 \\
\hline \multicolumn{4}{|l|}{ Siblings $(n=123)$} \\
\hline Number & 102 & 21 & \\
\hline Sex: males/females & $47 / 55$ & $6 / 15$ & 0.11 \\
\hline Age: mean (SD), years & $36.9 \mid(10.1)$ & $35.5 \mid(10.6)$ & 0.56 \\
\hline Education: mean (SD), years & $16.0 \mid(2.3)$ & $15.5 \mid(2.2)$ & 0.35 \\
\hline Handedness: mean (SD) & $78.0 \mid(47.8)$ & $87.6 \mid(41.5)$ & 0.39 \\
\hline IQ: mean (SD) & $106.7 \mid(10.0)$ & $108.1 \mid(12.1)$ & 0.59 \\
\hline 2-back correct: mean (SD), \% & $76 \mid(20)$ & $77 \mid(13)$ & 0.81 \\
\hline 2-back RT: mean (SD), seconds & $0.53 \mid(0.28)$ & $0.54 \mid(0.26)$ & 0.86 \\
\hline \multicolumn{4}{|l|}{ Patients ( $n=78$ ) } \\
\hline Number & 64 & 14 & \\
\hline Sex: males/females & $54 / 10$ & $10 / 4$ & 0.22 \\
\hline Age: mean (SD), years & $32.8 \mid(9.7)$ & $32.1 \mid(9.9)$ & 0.79 \\
\hline Education: mean (SD), years & $14.5 \mid(2.4)$ & $14.7 \mid(2.6)$ & 0.72 \\
\hline Handedness: mean (SD) & $73.7 \mid(58.5)$ & $96.4 \mid(7.5)$ & 0.15 \\
\hline IQ: mean (SD) & $95.5 \mid(11.3)$ & $88.7 \mid(10.7)$ & 0.04 \\
\hline 2-back correct: mean (SD), \% & $69 \mid(20)$ & $70 \mid(18)$ & 0.74 \\
\hline 2-back RT: mean (SD), seconds & $0.61 \mid(0.24)$ & $0.63 \mid(0.29)$ & 0.79 \\
\hline CPZEs: mean (SD) & $490.1 \mid(435.5)$ & $477.9 \mid(468.3)$ & 0.91 \\
\hline Positive symptoms (PANSS): mean (SD) & $12.3 \mid(6.1)$ & $13.9 \mid(5.4)$ & 0.54 \\
\hline Negative symptoms (PANSS): mean (SD) & $19.2 \mid(10.0)$ & $16.9 \mid(9.4)$ & 0.28 \\
\hline Delusion severity (PANSS): mean (SD) & $3.0 \mid(2.1)$ & $2.9 \mid(1.7)$ & 0.64 \\
\hline Global functioning (GAF): mean (SD) & $50.5 \mid(14.1)$ & $52.9 \mid(11.5)$ & 0.38 \\
\hline
\end{tabular}

RT, Reaction time; GAF, Global Assessment of Functioning; PANSS, Positive and Negative Symptom Scale.

diagnosis interaction effects, we tested an ANCOVA model with diagnosis (control, sibling, patient) and NRG3 genotype (T/T homozygotes, $\mathrm{C}$ allele carriers) as factors of interest and age, sex, $n$-back task performance, and IQ as nuisance covariates.

Statistical inference. Prior evidence with this task suggests a relatively stronger "inefficiency signaling" in the DLPFC of the right hemisphere in schizophrenia patients and individuals at genetic risk for the disorder (for review, see Rasetti and Weinberger, 2011). Accordingly, significance was measured at $p<0.05$ family-wise error (FWE) corrected for multiple comparisons at the voxel level in an a priori defined anatomical mask of the right DLPFC derived from the Wake Forest University Pickatlas toolbox (http://www.fmri.wfubmc.edu). The mask included cortical gray matter areas that corresponded to the equivalents of BA 9 and 46 in the MNI template space. Outside this prehypothesized region of interest (ROI), findings were considered significant if they passed a significance threshold of $p<0.05$ FWE corrected for multiple comparisons across the whole brain.

\section{Results}

There were no differences in performance accuracy or reaction time based on genotype for any of the diagnostic groups of subjects (Table 1). Thus, physiologic differences presumably reflect how the cognitive information is handled in brain and not how individuals perform on the task. Consistent with prior studies, we detected effects of increased genetic risk in healthy siblings in the contrast "controls < siblings" that conform to established models of prefrontal inefficiency linked to genetic risk for schizophrenia (MNI: $x=48, y=0, z=42 ; T=3.2 ; P_{\mathrm{FWE}}=0.1$, corrected within DLPFC ROI). The locales within DLPFC showing this effect are similar to those highlighted in previous studies. While this effect closely approached but did not survive stringent familywise error correction in this sample, given the ample prior evidence for this association, the adopted significance threshold is likely excessive.

Our first test of genotype involved the effects of rs 10748842 on prefrontal cortical physiology in healthy individuals. We observed highly significant and regionally specific effects of NRG3 genotype on right VLPFC function, whereby during working memory, greater activation was observed in rs10748842 minor allele (C) carriers compared with risk-associated $\mathrm{T}$ allele homozygotes (MNI: $x=39, y=33, z=-3 ; T=4.8 ; P_{\mathrm{FWE}}=0.008$, whole-brain corrected; Fig. $1 A$ ). This finding was counter to our hypothesis. We then performed an analysis that included all groups, i.e., healthy controls, unaffected siblings, and patients with schizophrenia. Whereas this combined group analysis did not identify a main effect of genotype, it revealed a highly significant genotype by group interaction in right DLPFC physiology in a separate locale (MNI: $x=33, y=39, z=15 ; T=4.6$; $P_{\mathrm{FWE}}=0.016$, whole-brain corrected; Fig. $1 C$ ). The extracted peak signals in right DLPFC (Fig. $1 B$ ) suggested relatively greater activation in $\mathrm{C}$ allele carriers in the siblings and healthy volunteers, an allele association that appeared to be reversed in patients.

An important factor that may complicate the interpretation of fMRI-based working memory data is task performance, as prefrontal cortical underactivation is, in general, associated with poor performance (Callicott et al., 2003b). Thus, it is conceivable that the divergent directionality of genotype association is be- 

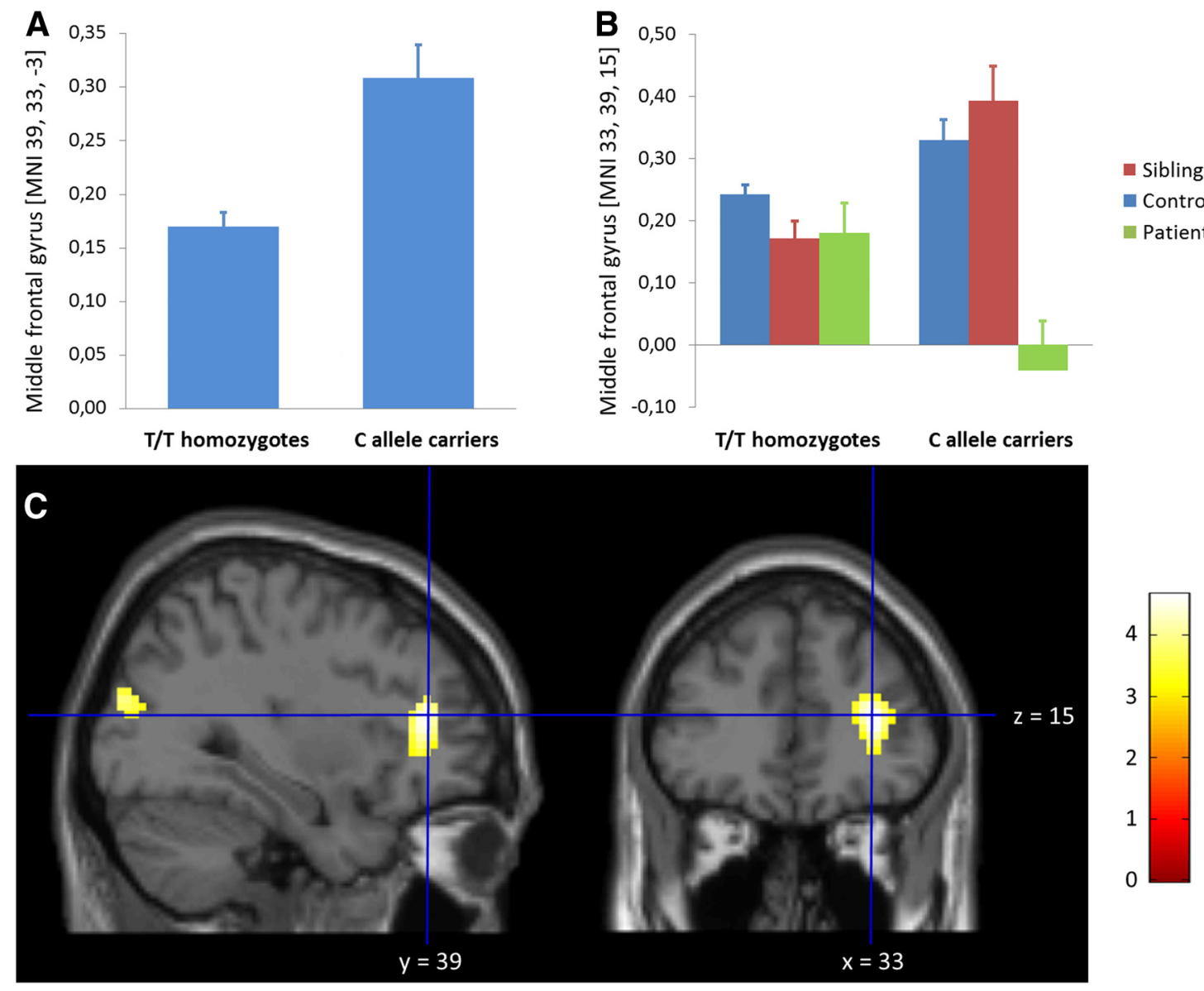

Figure 1. Effects of NRG3 rs 10748842 on prefrontal cortex physiology during working memory performance. $A$, Significantly greater activation of the VLPFC during working memory performance in healthy volunteers carrying one or two C alleles compared with T/T homozygotes during 2-back performance relative to 0 -back performance $(Z=4.7 ; p=0.008$, whole-brain FWE corrected). $B$, Significant group by NRG3 genotype interaction effects on DLPFC activation during working memory performance $(Z=4.6 ; p=0.016$, whole-brain FWE corrected). The bars represent means across subjects, and the error bars represent SEM. C, The activation map of the group by genotype interaction analysis is overlaid on sagittal and coronal sections of a structural template image ( $p=$ 0.001 , uncorrected, for presentation purposes). The color bar represents $t$ values. Notably, the unstandardized effect sizes in $\boldsymbol{A}$ and $\boldsymbol{B}$ are at the order of $0.1 \%$ whole-brain mean changes in BOLD signal, indicating that the large sample size did not sensitize our analyses to trivial effect sizes.

cause of the poorer performance in the patients. However, supplementary analyses in a total sample of $n=395$ subjects with performance-balanced diagnosis and genotype subgroups $\left(F_{(2,394)}<0.6, p>0.5\right)$ suggested that the observed directionality of NRG3 allele effects in DLPFC is not explained by differences in task performance (MNI: $x=36, y=39, z=12 ; T=3.6 ; P_{\mathrm{FWE}}=$ 0.04 , corrected within DLPFC ROI). Also, no differential correlation between prefrontal activity and behavioral performance across genotypes and across diagnostic groups were observed.

Finally, as striking as the genotype effects appear to be, analyses of BOLD signals in the locations in VLPFC and DLPFC showing NRG3 genotype effects did not provide any evidence for a corresponding effect of diagnosis (i.e., prefrontal inefficiency) in the healthy siblings of schizophrenia patients relative to controls ( $p \geq 0.05$, uncorrected) (Callicott et al., 2003a; Rasetti and Weinberger, 2011). This suggests that the genotype effects on prefrontal function, albeit highly significant in their own right, are not directly linked to the $n$-back-related neurophysiologic phenotype that is linked to increased genetic risk for schizophrenia in these subjects.

\section{Discussion}

In this study, we provide novel evidence that a schizophrenia risk-associated polymorphism in NRG3 (rs10748842) impacts prefrontal cortical physiology during working memory performance. We had predicted that, like a number of other schizophrenia risk-associated genes, NRG3 would show association with a variation in prefrontal cortical physiology (i.e., inefficiency) that also has been linked with an increased genetic risk for schizophrenia. That is, we expected that individuals carrying riskassociated genotypes would show less efficient engagement (i.e., hyperactivation for a given level of performance) of DLPFC than other genotype groups. Although we found evidence of this predicted association in a patient sample, we did not find this effect in healthy subjects or in healthy siblings of patients, who showed opposite allelic associations. It is this difference that constitutes the genotype by group interaction. Moreover, none of these genetic associations mapped to locales within prefrontal cortex that themselves were associated with increased genetic risk for schizophrenia (i.e., showing greater activation in siblings than controls matched for performance). In other words, whereas we report strong NRG3 association with prefrontal cortical physiology, we cannot conclude that this is the neurophysiological mechanism of the association of NRG3 with schizophrenia.

Specifically, we observed a whole-brain significant increase in activation in VLPFC in healthy control carriers of the rs 10748842 nonrisk C allele. Given that a sole analysis of control individuals 
runs the risk of identifying allele effects that have little or no relationship to the disorder itself, we also conducted a secondary analysis in which we included controls, schizophrenia patients, and their unaffected siblings. Here, we observed a whole-brain significant genotype by group interaction effect in DLPFC suggestive of a relative activation increase in healthy volunteers and healthy siblings carrying the nonrisk $\mathrm{C}$ allele, but a relative activation decrease in patients carrying the $C$ allele. Although this result is statistically compelling, the prefrontal cortical region involved is not the same locale in which siblings show greater inefficiency than controls. Recall that performance was balanced between genotype groups or was used as a nuisance variable and can thus (in principle) not be invoked to explain these effects.

These inconsistencies notwithstanding, our data provide novel evidence that a schizophrenia-associated polymorphism in NRG3, which has previously shown molecular association to NRG3 mRNA splice isoform expression in PFC, has a strong impact on PFC physiology during working memory performance. Previous associations to disease status, delusion, and positive symptom severity have been reported in this patient population to the T allele (Kao et al., 2010). However, in controls and siblings, who performed normally on the task, the observation of decreased engagement of DLPFC and VLPFC in T/T individuals compared with $\mathrm{C}$ allele carriers suggests that the $\mathrm{T} / \mathrm{T}$ genotype is associated with a more efficient prefrontal physiology, an unexpected result compared with many risk genes studied with fMRI during working memory in healthy subjects. However, opposite directionalities of genetic associations in patients compared with controls have been reported (Meyer-Lindenberg et al., 2007; Bertolino et al., 2009), but the interpretation is particularly complicated here, because the extracted analysis revealed a significantly reduced DLPFC engagement in the patient group (i.e., "hypofrontality," not "inefficiency") compared with the other two groups. Whereas hypofrontality has generally been related to poorer task performance in patients (Callicott et al., 2003b), our supplementary analyses in performance-balanced subgroups suggests that differences in task behavior do not explain the variant directionality of allele effects in patients.

Another consideration is the possibility that the associations of NRG3 with schizophrenia and with prefrontal physiology are orthogonal. For example, although we replicated prior evidence for a classical intermediate phenotype physiology within DLPFC (i.e., healthy siblings showing relative cortical engagement intermediate between patients and controls), the same empirical link could not be established for the specific locations within DLPFC and VLPFC showing associations to NRG3 genotype. For this link, differences in BOLD response would be expected that follow the physiological pattern of a disproportionate activation increase in patients relative to controls, and an intermediate activation level in healthy siblings of patients, which was not evident in the areas showing NRG3 genotype effects in this study. Also, the observation that risk-associated genotypes are relatively more efficient in both controls and healthy siblings suggests that the schizophrenia risk-associated mechanism of NRG3 is not related to the prefrontal inefficiency intermediate phenotype, per se, which has been linked with a number of other risk-associated genes (Rasetti and Weinberger, 2011). Third, the observation that healthy siblings show similar genetic association as do unrelated normal control subjects suggests that the different direction of association in patients is related to state of illness factors not shared by the siblings, e.g., a potential interaction between NRG3 genetic variation and antipsychotic medication effects. Interestingly, the proposed genotype by medication interaction may also explain prior analogous reports at the cognitive-behavioral level where a protective effect of the minor allele was detected in medicated patients (Morar et al., 2011; Meier et al., 2013). Potentially consistent with our observations, a similar flip in allele effects was observed in healthy controls (Morar et al., 2011), whereby the minor nonrisk allele was associated with poorer cognitive performance. Although the neural system link between NRG3 and risk for schizophrenia may not be based on prefrontal cortical physiologic inefficiency, other risk-associated imaging phenotypes [e.g., hippocampal-prefrontal coupling (Rasetti et al., 2011), cingulate activation (Sambataro et al., 2013), or hippocampal activation (Rasetti et al., 2014)] may be more informative. These are subjects of future studies.

There is an additional but more speculative explanation for our findings that merits comment. Our results raise the question of whether a genetic advantage in normal individuals may translate into a disadvantage in the context of other genetic factors or disease-associated abnormalities, including abnormal prefrontal cortical function in schizophrenia. This explanation has been offered for similar dissociable genetic effects in other schizophreniarelated genes (Meyer-Lindenberg et al., 2007). However, we have no data to further illuminate this possibility.

In summary, we present evidence implicating NRG3 in brain processes related to schizophrenia and demonstrate that NRG3 genetic variation impacts neural function in human brain. However, the link between the effect of this variation in NRG3 on prefrontal function and its role as a schizophrenia risk factor is likely complex and related to a presently undefined neural systems-level mechanism. Our data underscore a crucial but often overlooked conceptual distinction in psychiatric imaging genetics: the observation of a statistical association between a genetic risk variant and a plausible neuroimaging phenotype alone is not sufficient to establish a convincing empirical link between this variant and the underlying neural mechanisms that translate the genetic risk for the disease into complex disease phenotypes (Rasetti and Weinberger, 2011).

\section{References}

Aron AR, Fletcher PC, Bullmore ET, Sahakian BJ, Robbins TW (2003) Stopsignal inhibition disrupted by damage to right inferior frontal gyrus in humans. Nat Neurosci 6:115-116. CrossRef Medline

Balciuniene J, Feng N, Iyadurai K, Hirsch B, Charnas L, Bill BR, Easterday MC, Staaf J, Oseth L, Czapansky-Beilman D, Avramopoulos D, Thomas GH, Borg A, Valle D, Schimmenti LA, Selleck SB (2007) Recurrent 10q22-q23 deletions: a genomic disorder on 10q associated with cognitive and behavioral abnormalities. Am J Hum Genet 80:938-947. CrossRef Medline

Bertolino A, Fazio L, Caforio G, Blasi G, Rampino A, Romano R, Di Giorgio A, Taurisano P, Papp A, Pinsonneault J, Wang D, Nardini M, Popolizio T, Sadee W (2009) Functional variants of the dopamine receptor D2 gene modulate prefronto-striatal phenotypes in schizophrenia. Brain 132:417425. CrossRef Medline

Callicott JH, Egan MF, Mattay VS, Bertolino A, Bone AD, Verchinksi B, Weinberger DR (2003a) Abnormal fMRI response of the dorsolateral prefrontal cortex in cognitively intact siblings of patients with schizophrenia. Am J Psychiatry 160:709-719. CrossRef Medline

Callicott JH, Mattay VS, Verchinski BA, Marenco S, Egan MF, Weinberger DR (2003b) Complexity of prefrontal cortical dysfunction in schizophrenia: more than up or down. Am J Psychiatry 160:2209-2215. CrossRef Medline

Carteron C, Ferrer-Montiel A, Cabedo H (2006) Characterization of a neural-specific splicing form of the human neuregulin 3 gene involved in oligodendrocyte survival. J Cell Sci 119:898-909. CrossRef Medline

Chen PL, Avramopoulos D, Lasseter VK, McGrath JA, Fallin MD, Liang KY, Nestadt G, Feng N, Steel G, Cutting AS, Wolyniec P, Pulver AE, Valle D (2009) Fine mapping on chromosome 10q22-q23 implicates Neuregulin 3 in schizophrenia. Am J Hum Genet 84:21-34. CrossRef Medline 
Egan MF, Straub RE, Goldberg TE, Yakub I, Callicott JH, Hariri AR, Mattay VS, Bertolino A, Hyde TM, Shannon-Weickert C, Akil M, Crook J, Vakkalanka RK, Balkissoon R, Gibbs RA, Kleinman JE, Weinberger DR (2004) Variation in GRM3 affects cognition, prefrontal glutamate, and risk for schizophrenia. Proc Natl Acad Sci U S A 101:12604-12609. CrossRef Medline

Fallin MD, Lasseter VK, Wolyniec PS, McGrath JA, Nestadt G, Valle D, Liang KY, Pulver AE (2003) Genomewide linkage scan for schizophrenia susceptibility loci among Ashkenazi Jewish families shows evidence of linkage on chromosome 10q22. Am J Hum Genet 73:601-611. CrossRef Medline

Faraone SV, Hwu HG, Liu CM, Chen WJ, Tsuang MM, Liu SK, Shieh MH, Hwang TJ, Ou-Yang WC, Chen CY, Chen CC, Lin JJ, Chou FH, Chueh CM, Liu WM, Hall MH, Su J, Van Eerdewegh P, Tsuang MT (2006) Genome scan of Han Chinese schizophrenia families from Taiwan: confirmation of linkage to 10q22.3. Am J Psychiatry 163:1760-1766. CrossRef Medline

Hariri AR, Weinberger DR (2003) Imaging genomics. Br Med Bull 65:259_ 270. CrossRef Medline

Huffaker SJ, Chen J, Nicodemus KK, Sambataro F, Yang F, Mattay V, Lipska BK, Hyde TM, Song J, Rujescu D, Giegling I, Mayilyan K, Proust MJ, Soghoyan A, Caforio G, Callicott JH, Bertolino A, Meyer-Lindenberg A, Chang J, Ji Y, et al. (2009) A primate-specific, brain isoform of KCNH2 affects cortical physiology, cognition, neuronal repolarization and risk of schizophrenia. Nat Med 15:509-518. CrossRef Medline

Kao WT, Wang Y, Kleinman JE, Lipska BK, Hyde TM, Weinberger DR, Law AJ (2010) Common genetic variation in Neuregulin 3 (NRG3) influences risk for schizophrenia and impacts NRG3 expression in human brain. Proc Natl Acad Sci U S A 107:15619-15624. CrossRef Medline

Koechlin E, Ody C, Kouneiher F (2003) The architecture of cognitive control in the human prefrontal cortex. Science 302:1181-1185. CrossRef Medline

Meier S, Strohmaier J, Breuer R, Mattheisen M, Degenhardt F, Muhleisen TW, Schulze TG, Nothen MM, Cichon S, Rietschel M, Wust S (2013) Neuregulin 3 is associated with attention deficits in schizophrenia and bipolar disorder. Int J Neuropsychopharmacol 16:549-556. CrossRef Medline

Meyer-Lindenberg A, Straub RE, Lipska BK, Verchinski BA, Goldberg T, Callicott JH, Egan MF, Huffaker SS, Mattay VS, Kolachana B, Kleinman JE, Weinberger DR (2007) Genetic evidence implicating DARPP-32 in human frontostriatal structure, function, and cognition. J Clin Invest 117:672-682. CrossRef Medline

Morar B, DragoviæM, Waters FA, Chandler D, Kalaydjieva L, Jablensky A (2011) Neuregulin 3 (NRG3) as a susceptibility gene in a schizophrenia subtype with florid delusions and relatively spared cognition. Mol Psychiatry 16:860-866. CrossRef Medline

Nixon DC, Prust MJ, Sambataro F, Tan HY, Mattay VS, Weinberger DR, Callicott JH (2011) Interactive effects of DAOA (G72) and catechol-Omethyltransferase on neurophysiology in prefrontal cortex. Biol Psychiatry 69:1006-1008. CrossRef Medline

Rasetti R, Weinberger DR (2011) Intermediate phenotypes in psychiatric disorders. Curr Opin Genet Dev 21:340-348. CrossRef Medline

Rasetti R, Sambataro F, Chen Q, Callicott JH, Mattay VS, Weinberger DR (2011) Altered cortical network dynamics: a potential intermediate phenotype for schizophrenia and association with ZNF804A. Arch Gen Psychiatry 68:1207-1217. CrossRef Medline

Rasetti R, Mattay VS, White MG, Sambataro F, Podell JE, Zoltick B, Chen Q, Berman KF, Callicott JH, Weinberger DR (2014) Altered hippocampalparahippocampal function during stimulus encoding: a potential indicator of genetic liability for schizophrenia. JAMA Psychiatry, in press.

Sambataro F, Mattay VS, Thurin K, Safrin M, Rasetti R, Blasi G, Callicott JH, Weinberger DR (2013) Altered cerebral response during cognitive control: a potential indicator of genetic liability for schizophrenia. Neuropsychopharmacology 38:846-853. CrossRef Medline

Tan HY, Sust S, Buckholtz JW, Mattay VS, Meyer-Lindenberg A, Egan MF, Weinberger DR, Callicott JH (2006) Dysfunctional prefrontal regional specialization and compensation in schizophrenia. Am J Psychiatry 163 1969-1977. CrossRef Medline

van Erp TG, Guella I, Vawter MP, Turner J, Brown GG, McCarthy G, Greve DN, Glover GH, Calhoun VD, Lim KO, Bustillo JR, Belger A, Ford JM, Mathalon DH, Diaz M, Preda A, Nguyen D, Macciardi F, Potkin SG (2013) Schizophrenia miR-137 locus risk genotype is associated with dorsolateral prefrontal cortex hyperactivation. Biol Psychiatry. Advance online publication. Retrieved Aug. 1, 2013. doi:10.1016/j.biopsych.2013.06.016. CrossRef Medline

Xu B, Woodroffe A, Rodriguez-Murillo L, Roos JL, van Rensburg EJ, Abecasis GR, Gogos JA, Karayiorgou M (2009) Elucidating the genetic architecture of familial schizophrenia using rare copy number variant and linkage scans. Proc Natl Acad Sci U S A 106:16746-16751. CrossRef Medline 